\title{
Dräktförändring hos en intersex gräsand Anas platyrhynchos över en sexårsperiod
}

\author{
Plumage changes in an intersex Mallard \\ Anas platyrhynchos over a six-year period
}

\author{
Ann-Katrin "AX" Sjögren' \& Jonas Waldenström² iD \\ 'Gluntens väg 8, 90737 Umeå, Sweden | ax@axart.se 22 Department of Biology and Environmental Science, Linnaeus \\ University, 39182 Kalmar, Sweden | jonas.waldenstrom@Inu.se
}

\begin{abstract}
INTERSEX INDIVIDUALS with a mixture of female and male plumage traits are reported regularly, but at low frequency. Here we describe the changes in plumage in a Mallard Anas platyrhynchos intersex individual over six years (2015-2020), from a mixed female-male phenotype to a predominantly male phenotype. Already at the first observation, typical male characteristics were present, such as greyish-white tail with two central curved black rectrices and a greenish tinge to parts of the head. The bird attained a more male-like plumage with the next moult and maintained that plumage for the rest of the study period. However, presence of female-patterned body feathers continued until 2019, but in reduced frequency, except for the cheeks and ear-coverts, which displayed a variation in colour. The bill remained female-patterned throughout and the bird did not enter eclipse plumage.
\end{abstract}

\section{Keywords: Anatidae | duck | field studies | gradual masculinization | longitudinal observations | phenotype | photographic documentation}

\section{Inledning}

Hos fåglar kan man sällsynt se individer som uppvisar en blandning av hon- och hankaraktärer, så kallade intersex. Fåglar i sådan dräkt kan misstas för hybrider, inte minst då hybridisering är relativt vanligt före- kommande hos exempelvis änder, särskilt från parker, zoon och annan fågeluppfödning där olika arter hålls tillsammans (Johnsgard 1960, Reeber 2015). Även om faglar med intersexutseende då och då rapporteras $\mathrm{i}$ 
fält är beskrivningarna sällan ingående (men se Chiba \& Honma 2011 för en genomgång av intersex hos stjärtand Anas acuta). I den här notisen beskrivs den gradvisa utvecklingen av intersex hos en gräsand Anas platyrhynchos som följts av en av oss (AX) under sex års tid i Umeå, Västerbotten. Fågeln finns fortfarande där när detta skrivs i januari 2021.

\section{Dokumentation}

Den första observationen skedde 1 april 2015 vid Lasarettsdammen vid Umeå Universitetssjukhus i centrala Umeå. Fågeln var vid tillfället inne på åtminstone sitt andra kalenderår, det vill säga kläckt 2014 eller tidigare. Under efterföljande år har fågeln regelbundet observerats från slutet av sommaren och hela vinterhalvåret (fram till april) i området kring dammen, där också fågelmatning sker. Under sommaren finns färre observationer, men den har noterats bland annat på Umeälven i staden vilket gör det troligt att den finns i staden året runt. Vid olika tillfällen har fågeln fotograferats och dessa bilder möjliggör en beskrivning av dräktutvecklingen över tid (tabell 1).

\section{DRÄKTUTVECKLING}

Våren 2015 uppvisar fågeln en dräkt med tydlig blandning av hon- och hantecknade fjädrar (figur 1). Ett första intryck ger en mestadels hontecknad dräkt, med typisk näbb och med stort inslag av hontecknade bruna, kamouflerande kroppsfjädrar, framförallt på kroppssidorna men även delvis på bröst och mantel. Även huvudet är mestadels honlikt, men med viss grönton på hjässa och nacke; dock finns ett tydligt streck genom ögonpartiet och mestadels hontecknade, ljust brunaktiga kinder och örontäckare. Tertialerna däremot är hantecknade och även stjärten uppvisar hanteckning med mestadels gråvita stjärtpennor, samt de två böjda svarta stjärtfjädrarna som är karakteristiska för gräsandshanar (Reeber 2015, Andersson et al. 2016). Bröstets teckning har en mörk kastanjebrun bottenfärg som är gängse hos hanar, men upplevs melerat och inte tydligt avsatt mot kroppssidorna.

Nästföljande vintersäsong (2015-2016), efter en ruggning under sommaren, börjar fågeln uppvisa en huvudsakligen hanlik dräkt (figur 2). Förändringen innebär att de hontecknade fjädrarna i huvudet blivit färre och att ett större grönt fjäderparti tillkommit,

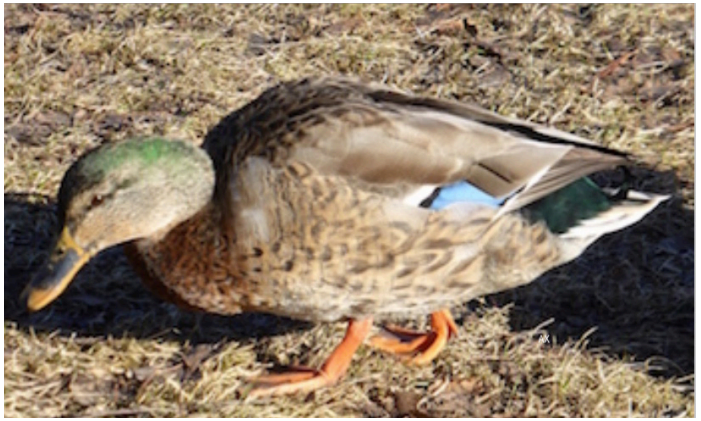

FIGUR 1. Vid första dokumentationen uppvisar intersexgräsanden Anas platyrhynchos en blandad dräkt, med både tydligt hontecknade fjädrar (på kroppsida, bröst, mantel, rygg) och hantecknade fjädrar (tertialer, vingspegel, stjärt) samt en lätt grönton på del av huvudet. Näbben hontecknad. Bild tagen 8 april 2015. Foto: Ann-Katrin Sjögren. - At the first observation, 8 April 2015, the intersex Mallard Anas platyrhynchos shows a clear mix of female and male plumage traits. Female-patterned feather tracts include sides of the body, parts of the breast, mantle and back, while tertials and tail have male-like traits. Moreover, the bird has a greenish tinge to parts of the head. Bill female-patterned. Photo: Ann-Katrin Sjögren.

främst på övre delen av huvudet, från näbbroten och ögat upp till hjässan, samt bakåt nacken. Gröntonen i det partiet är inte homogen, och uppfattas olika beroende på ur vilken vinkel fotografiet är taget. Det kastanjebruna bröstet har fått mindre inslag av melering och är tydligare avgränsat mot kroppssidorna, vilka också antagit en mer hanlik karaktär, med ljust grå färg. Dock finns fortfarande inslag av hontecknade fjädrar på kroppssidorna. Även mantel och rygg är mestadels hantecknade, även om dessa partier upplevs som mindre distinkt och mindre kontrastrikt tecknade än dräkten hos genetiska hanar.

Under efterföljande vintrar blir dräkten mer distinkt. I grund och botten är nu fägeln fenotypiskt en hane, med gråa kroppssidor, kastanjebrunt bröst, hantecknade skapularer och tertialer, tydlig blå vingspegel och en hanstjärt (figur 3). Enstaka hontecknade fjädrar finns på kroppssidorna fram till vintern 2019-2020, därefter är även dessa helt hantecknade. Kvarvarande variation återfinns i huvudteckningen, där kindens och örontäckarnas färg varierar från vitaktig till mestadels grön, ibland endast glest fjäderbeväxt. Den enda tydligt honlika karaktären är näbben, som genom hela studieperioden är hontecknad med stort mörkt mittparti i stället för en hanes typiska gröngula näbb (Reeber 2015, Andersson et al. 2016).

Under sommar och tidig höst finns färre fotografier, 
TABELL 1 (höger). Datum och länkar (URL:er) till alla fotografier av intersexgräsanden Anas platyrhynchos i Artportalen.

- (right). Dates (YYYY-MM-DD) and links (URLs) to all photographies of the intersex Mallard Anas platyrhynchos deposited in the Swedish Species Observation System.

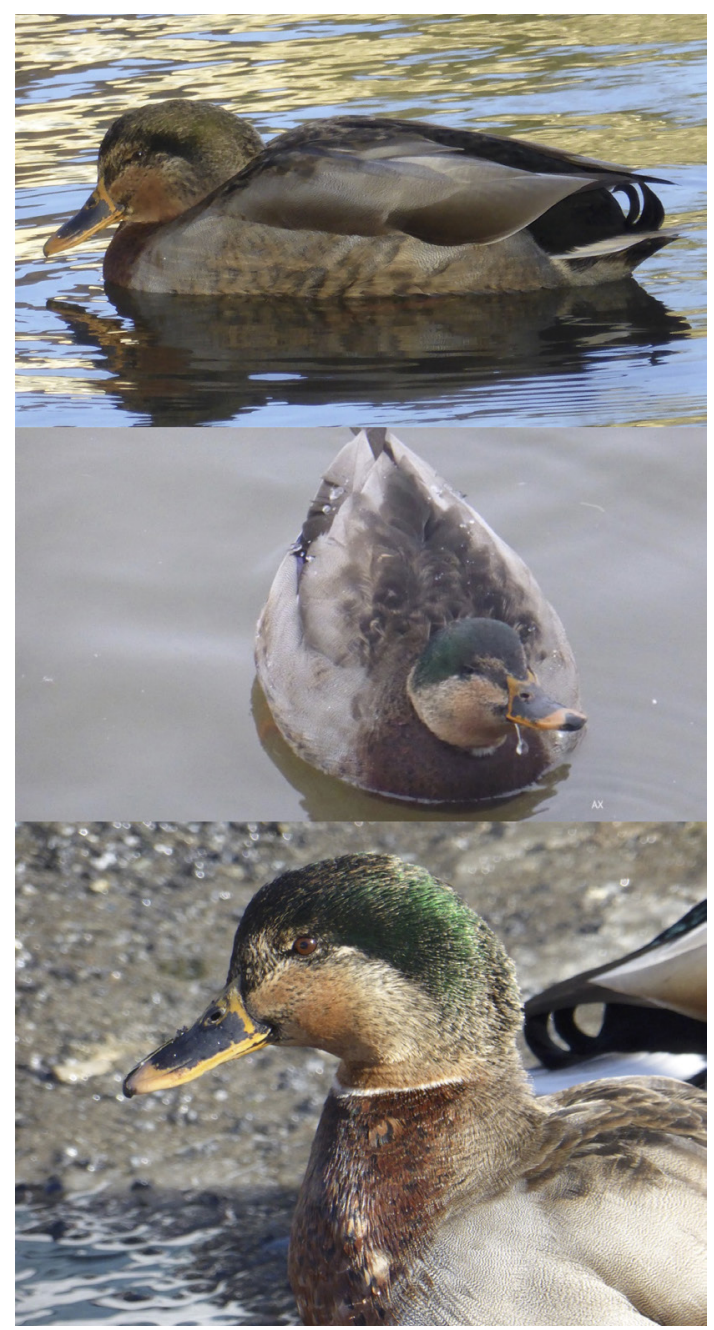

FIGUR 2. Vintern 2015/2016 har dräkten antagit en alltmer hanlik karaktär, med mer grönt inslag på hjässa och nacke, gråare kroppssidor (dock med förekomst av hontecknade fjädrar), kastanjebrunt något melerat bröst, rygg och skapularer mer hantecknade, samt fortsatt hantecknad vinge och stjärt. Bilder (uppifrån och ner) tagna 4 november 2015, 24 januari 2016 och 15 mars 2016. Foto: Ann-Katrin Sjögren. - In winter 2015/2016 the bird has a more pronounced male-like plumage, with larger extent of green on head and neck, generally grey sides of the body (but with occurrence of female-patterned feathers), chestnut brown breast, mostly male-patterned back and scapulars, and a male wing and tail. Photos from 4 November 2015 (top), 24 January 2016 (middle), and 15 March 2016 (bottom). Photo: Ann-Katrin Sjögren.
Datum Date URL

2015-04-08 https://www.artportalen.se/Image/1368030

2015-04-11 https://www.artportalen.se//mage/1368354

2015-11-04 https://www.artportalen.se/lmage/1479287

2015-11-13 https://www.artportalen.se//mage/1482206

2016-01-24 https://www.artportalen.se//mage/1503868

2016-03-15 https://www.artportalen.se/Image/1521378

2016-03-15 https://www.artportalen.se/Image/1521379

2016-03-29 https://www.artportalen.se/Image/1527475

2016-08-17 https://www.artportalen.se/Image/1634395

2016-09-02 https://www.artportalen.se/Image/1646904

2016-09-21 https://www.artportalen.se/Image/1658807

2016-11-18 https://www.artportalen.se/Image/1687828

2016-11-19 https://www.artportalen.se/Image/1688297

2016-11-19 https://www.artportalen.se/Image/1688298

2017-01-30 https://www.artportalen.se/Image/1715134

2017-03-11 https://www.artportalen.se//mage/1729831

2017-08-08 https://www.artportalen.se/Image/1886912

2017-10-24 https://www.artportalen.se/Image/1961513

2018-01-26 https://www.artportalen.se/Image/2005581

2018-08-15 https://www.artportalen.se/Image/2175625

2018-08-28 https://www.artportalen.se/Image/2187098

2018-10-28 https://www.artportalen.se/Image/2241975

2019-01-07 https://www.artportalen.se/Image/2279975

2019-01-29 https://www.artportalen.se//mage/2290527

2019-09-02 https://www.artportalen.se/Image/2543790

2019-09-18 https://www.artportalen.se/Image/2565345

2019-11-13 https://www.artportalen.se//mage/2622888

2019-12-08 https://www.artportalen.se/Image/2637648

2020-01-03 https://www.artportalen.se/Image/2656254

2020-01-16 https://www.artportalen.se/Image/2664807

2020-02-11 https://www.artportalen.se/Image/2686345

2020-03-12 https://www.artportalen.se/Image/2709691

2020-04-21 https://www.artportalen.se//mage/2787083

2020-07-25 https://www.artportalen.se//mage/2979507

2020-10-11 https://www.artportalen.se/Image/3110335

2020-10-23 https://www.artportalen.se/Image/3125971

2020-11-14 https://www.artportalen.se/Image/3151041

2020-11-22 https://www.artportalen.se/Image/3160153

2020-11-28 https://www.artportalen.se/Image/3165480

2020-12-02 https://www.artportalen.se/Image/3168950

2020-12-14 https://www.artportalen.se//mage/3177658

2020-12-18 https://www.artportalen.se/Image/3180642

2020-12-31 https://www.artportalen.se/Image/3190584

2021-01-27 https://www.artportalen.se/Image/3215819 
men även här ses en maskulinisering av dräkten över tid, med brokigare dräkt 2016 än vid senare observationer (figur 4). Det är dock oklart när ruggningen initieras i relation till andra gräsänder (vilken hos genetiska hanar är tidigare än för honor); bilderna är för fă för att ge tydlig ledning. En tydlig skillnad är att fågeln aldrig setts bära en honlik eklipsdräkt.

\section{BETEENDE}

Beteendemässigt har faggeln betett sig som andra gräsänder vid dammen med matning, där den oftast vilat tillsammans med gräsandshanar. Även sommartid, när den setts vid älven, har den sällskapat med hanar. Inga könsspecifika läten har hörts, utan endast enstaka kvackningar vissa gånger vid matning, men den har oftast varit tyst.

\section{Diskussion}

Vid första dokumentationstillfället var dräkten en tydlig blandning av hon- och hantecknade fjädrar, men redan ett halvår senare var dräkten i huvudsak hanlik. Efter detta skedde förändringen i dräkten långsammare och fortfarande efter fyra år sågs enstaka hontecknade fjädrar i fjäderdräkten. Den del av dräkten som uppvisade störst variation även under senare år var huvudteckningen, främst kinden och örontäckarna som varierade i färg både mellan vinter och sommar, och mellan år, ibland också med endast gles fjäderbeväxning.

Utveckling av den alltmer hanlika dräkten stämmer med kunskapsläget om könsförändringar hos änder och andra fåglar. Till skillnad från däggdjur är det hos fåglar honorna som är det heterogametiska könet, med könskromosomerna ZW, och hanarna det monogametiska könet, med könskromosomerna ZZ (Major \& Smith 2016, Smith 2010). Även om det är könskromosomerna som bestämmer det genetiska könet vid befruktningen är det produktionen av östrogen senare under utvecklingen som bestämmer honans fenotyp (Major \& Smith 2016). Hos faglar utvecklas bara ett av de två anlagen till en funktionell äggstock, och om denna skadas förändras produktionen av östrogen och en maskulinisering möjliggörs (Smith 2010). Förutom sjukdomar (exempelvis tumörer) och trauma (exempelvis skottskada) kan det tänkas att även hormonstörande

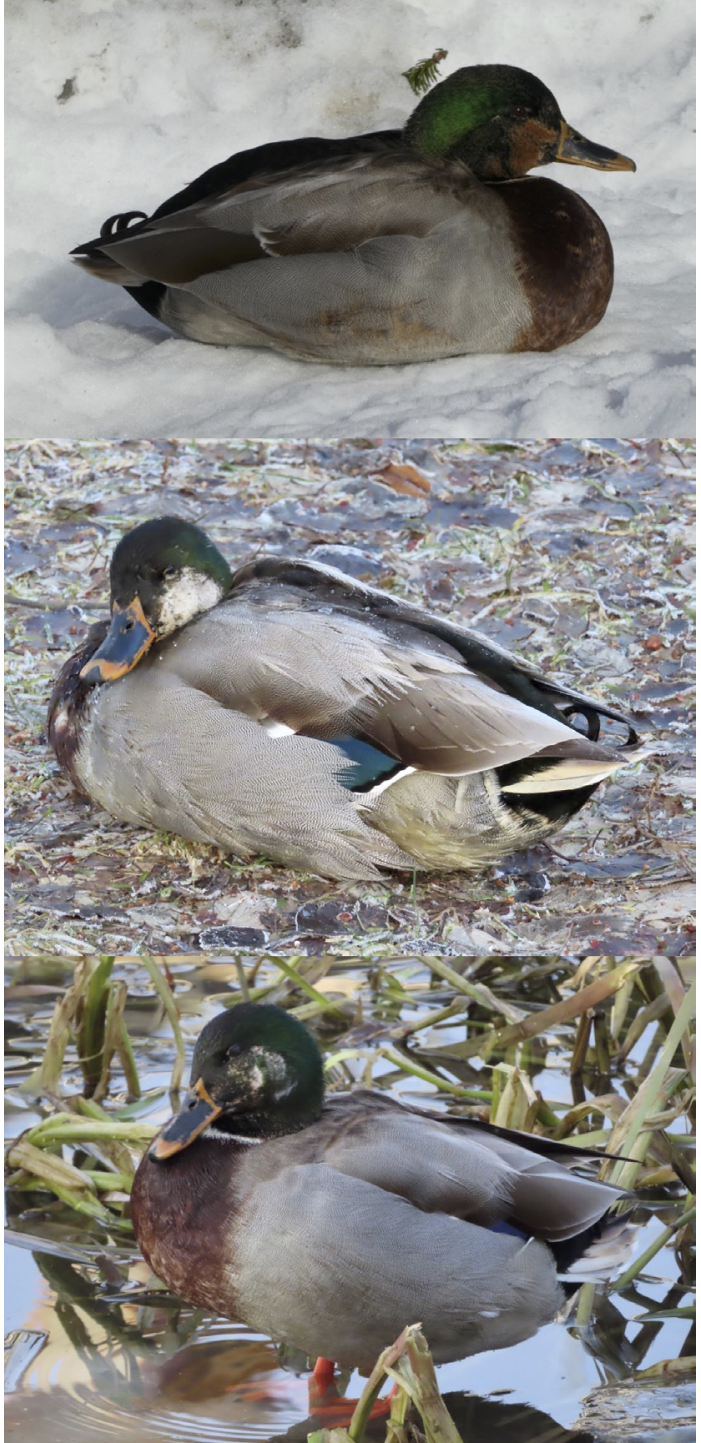

FIGUR 3. Efterföljande vintrar utvecklas intersexgräsandens Anas platyrhynchos dräkt långsammare och är genomgående mestadels hantecknad. Inslaget av hontecknade fjädrar på kroppsidorna försvinner alltmer. Huvudteckningen visar variation främst i kinden, som varierar från brun, till vit och grön. Observera hontecknad näbb på alla bilder. Bilder (uppifrån och ner) tagna 11 mars 2017, 8 december 2019 och 11 februari 2020. Foto: Ann-Katrin Sjögren.

- The following winters, the plumage changes in the intersex Mallard Anas platyrhynchos are slower and it appears mostly male-like in plumage. The extent of female-patterned body feathers decreases with time. The largest variation is seen in cheek and ear-coverts that vary between brown, white, and green. Photos (top to bottom) from 11 March 2017, 8 December 2019, and 11 February 2020. Photo: Ann-Katrin Sjögren. 


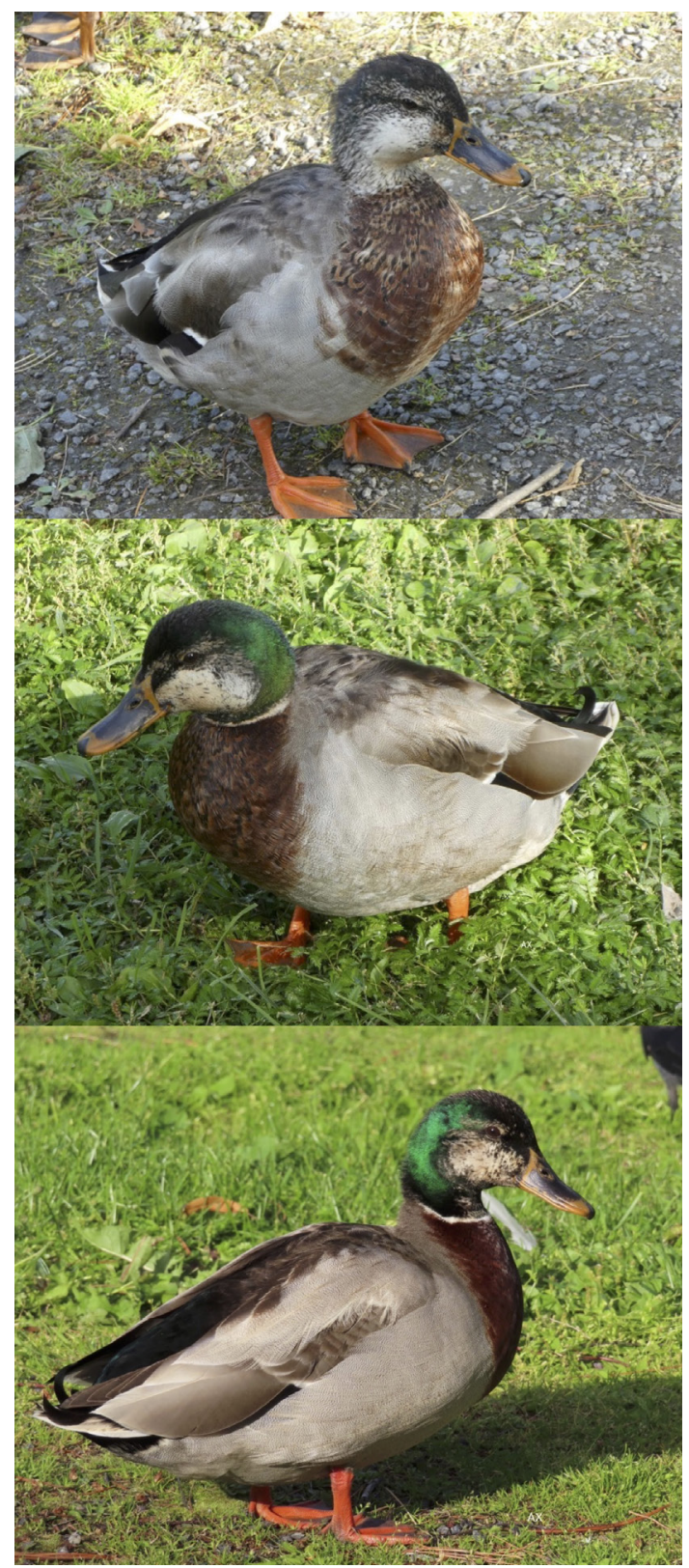

FIGUR 4. Observationer av intersexgräsanden Anas platyrhynchos från sommarhalvåret är färre, men även här utvecklas dräkten alltmer till hanlik dräkt. Fågeln tycks inte anlägga eklipsdräkt. Bilder (uppifrån och ner) tagna 2 september 2016, 18 augusti 2018 och 25 juli 2020. Foto: Ann-Katrin Sjögren.

- There are fewer observations during summer, but they also depict a bird with advancing male plumage. Interestingly, the intersex Mallard Anas platyrhynchos does not seem to attain eclipse plumage. Photos from 2 September 2016 (top), 18 August 2018 (middle) and 25 July 2020. Photo: Ann-Katrin Sjögren. kemikalier i miljön kan påverka äggstockens förmåga att producera östrogen.

Observationerna av gräsanden i Umeå antyder att förändringen skedde gradvis, men vi vet ingenting om den bakomliggande orsaken till uppkomsten av intersex i den här individen. Man kan dock spekulera $\mathrm{i}$ att händelsen som ledde till detta inträffat någon gång under 2014, eftersom de förändringarna av dräkten som sågs våren 2015 troligen anlagts vid ruggningen hösten innan. Oaktat orsaken till maskuliniseringen är fågeln i skrivande stund i sitt sjunde kalenderår, vilket visar att den klarat sig väl trots förändringen. Även om färre fotografier finns från sommar och tidig höst står det klart att fageln ej utvecklar traditionell eklipsdräkt som genetiska hanar gör.

Vid en studie av stjärtand på övervintringsplats $\mathrm{i}$ Japan beskrevs förekomsten av individer med intersexutseende till $0,01-0,18 \%$ under en tioårsperiod (i en population om $3800-8500$ stjärtänder; Chiba \& Honma 2011). Den studien visade också att intersex kan uppkomma både hos juvenila och adulta fåglar, och också med en variation mellan individer i graden av maskulinisering av fjäderdräkten. En stjärtand som följdes under flera vintrar uppvisade liknande dräktförändringar som gräsanden beskriven här, med en snabb förändring från hondräkt till intersex första året och därefter en långsammare maskulinisering (Chiba \& Honma 2011).

Om siffrorna över intersex hos stjärtand i Japan är överförbara till andra arter bör det finnas många individer med intersex även bland skandinaviska änder. Som tankeexempel: givet antagandet om att antal intersexindivider hos gräsand ligger i spannet o,o1-0,18\% och att antalet gräsänder är ca 200000 par (Wirdheim 2020), bör det finnas 40-720 intersex gräsänder i Sverige, eller en intersexindivid per 555-8 ooo gräsänder.

I tabell 2 sammanställs ett antal observationer av andra individer med intersexutseende som rapporterats med fotografi i det svenska rapportsystemet Artportalen (www.artportalen.se). De allra flesta av dessa är från gräsand och från ett fåtal observatörer, vilket indikerar att det finns ett mörkertal av observerade individer som inte rapporteras. Fåglarna i dessa bilder uppvisar en spännvidd av utseenden, från mestadels honlik till mestadels hanlik, och stämmer väl in på de faser som fågeln beskriven i denna uppsats visat över tid. Ett exempel med en ringmärkt fagel är extra intressant, då 
TABELL 2. Observationer i Sverige av intersex änder rapporterade i Artportalen. Länk anger bildnummer att läggas till URL:en https://www. artportalen.se/Image/.

- Observations in Sweden of intersex duck individuals reported in the Swedish Species Observation System. Dates are in the format YYYY-MM-DD. Link specifies image number, to add to the URL https://WwW.artportalen.se/Image/.

\begin{tabular}{|c|c|c|c|c|}
\hline $\begin{array}{l}\text { Plats } \\
\text { Location }\end{array}$ & $\begin{array}{l}\text { Observatör } \\
\text { Observer }\end{array}$ & $\begin{array}{l}\text { Datum } \\
\text { Date }\end{array}$ & $\begin{array}{l}\text { Länk } \\
\text { Link }\end{array}$ & $\begin{array}{l}\text { Kommentar } \\
\text { Comment }\end{array}$ \\
\hline \multicolumn{5}{|l|}{ Kricka Anas crecca Eurasian Teal } \\
\hline $\begin{array}{l}\text { Edebybron, Lövstaslätten, } \\
\text { Uppland }\end{array}$ & Nils-Olof Jerling & $2021-01-30$ & 3221523 & \\
\hline \multicolumn{5}{|c|}{ Gräsand Anas platyrhynchos Mallard } \\
\hline Jutebron, Kalmar & Simon Carrington & $\begin{array}{l}2019-01-19 \\
2019-01-27\end{array}$ & $\begin{array}{l}2286006 \\
2290307\end{array}$ & \\
\hline Stockholms ström, Uppland & Per Jakobsson & 2013-03-27 & 1230794 & \\
\hline Slottsparken, Malmö, Skåne & Carl Gunnar Gustavsson & 2013-01-21 & 1301894 & \\
\hline Stockholms ström, Uppland & Claes-Göran Magnusson & 2013-01-05 & 1218503 & \\
\hline Åkarp, Burlöv, Skåne & Carl Gunnar Gustavsson & 2012-10-29 & 1301899 & \\
\hline Slottsparken, Malmö, Skåne & Carl Gunnar Gustavsson & 2012-03-13 & 3224954 & \\
\hline Slottsparken, Malmö, Skåne & Carl Gunnar Gustavsson & $2012-02-21$ & 1134121 & \\
\hline Trelleborg, Skåne & Carl Gunnar Gustavsson & $2012-02-10$ & 1132017 & \\
\hline Stockholms ström, Uppland & Per Jakobsson & 2012-01-19 & 1127589 & \\
\hline $\begin{array}{l}\text { Delsjöområdet, Göteborg, } \\
\text { Västergötland }\end{array}$ & Leif Andersson & 2011-05-08 & 1052240 & \\
\hline Slottsparken, Malmö, Skåne & Carl Gunnar Gustavsson & 2010-12-12 & 1027241 & \\
\hline Gislövs läge, Skåne & Mattias Ullman & $2010-02-17$ & 956761 & \\
\hline Slottsparken, Malmö, Skåne & Carl Gunnar Gustavsson & 2010-01-10 & 949606 & \\
\hline $\begin{array}{l}\text { Pildammsparken, Malmö, } \\
\text { Skåne }\end{array}$ & Carl Gunnar Gustavsson & $2008-12-27$ & $\begin{array}{l}875925 \\
875929\end{array}$ & $\begin{array}{l}\text { Två till tre olika fåglar. } \\
\text { Two to three different individuals. }\end{array}$ \\
\hline Ortviken, Skön, Medelpad & Anders Andersson & 2008-02-12 & 826891 & \\
\hline $\begin{array}{l}\text { Pildammsparken, Malmö, } \\
\text { Skåne }\end{array}$ & Carl Gunnar Gustavsson & $\begin{array}{l}2007-04-15 \\
2007-12-24 \\
2008-01-19\end{array}$ & $\begin{array}{l}799155 \\
823276 \\
825210\end{array}$ & $\begin{array}{l}\text { Troligen samma individ baserat på } \\
\text { näbbteckning. } \\
\text { Most likely the same individual, based on } \\
\text { bill pattern. }\end{array}$ \\
\hline $\begin{array}{l}\text { Klippan, Göteborgs hamn, } \\
\text { Västergötland }\end{array}$ & Uno Unger & $\begin{array}{l}2007-01-18 \\
2009-11-16\end{array}$ & $\begin{array}{l}793220 \\
942569\end{array}$ & $\begin{array}{l}\text { Rehabiliterad och ringmärkt som adult } \\
\text { hona i september } 2002 \text {. Ring avläst i fält } \\
\text { i februari 2005, "såg då i princip ut som } \\
\text { en hane". } \\
\text { Ringed as adult female in September } 2002 . \\
\text { Male-like plumage from } 2005 \text { onwards. }\end{array}$ \\
\hline
\end{tabular}

den vid ringmärkningstillfället bedömdes som adult hona och tre år senare identifierades i fält med hjälp av ringnumret, men nu med en hanlik dräkt. En mer systematisk undersökning om frekvensen av intersex hos gräsand är önskvärd och vi vill därför avsluta med en uppmaning att rapportera observationer av intersex, exempelvis till Artportalen (www.artportalen.se).

\section{Tack}

Ett stort tack till de två granskarna som läst och kommenterat vårt manus. Ett extra stort tack skickar vi till Carl Gunnar Gustavsson som pushat för att denna uppsats skulle se dagens ljus, samt bidragit med bilder och kommentarer på intersexfåglar på Artportalen och via korrespondens. 


\section{Referenser}

Andersson S, Bengtsson D, Hellström M \& Waldenström J. 2016. Age and sex determination of Mallards Anas platyrhynchos in autumn. Ornis Svecica 26: 61-81. https://doi.org/10.34080/os.v26.22530

Chiba A \& Honma R. 2011. A study on the Northern Pintail (Anas acuta) females with masculinized plumage: their prevalence, morphological and behavioural traits, and reproductive organs. Journal of Ornithology 152: 733-742. https://doi.org/10.1007/ s10336-011-0654-9

Johnsgard PA. 1960. Hybridization in the Anatidae and its taxonomic implication. The Condor 62: 25-33. https://doi.org/10.2307/1365656
Major AT \& Smith CA. 2016. Sex reversal in birds. Sexual Development 10: 288-30o. https://doi.org/10.1159/000448365

Reeber S. 2015. Wildfowl of Europe, Asian and North America. Helm Identification Guides. Bloomsbury Publishing, London. 656 sid.

Smith CA. 2010. Sex determination in birds: a review. Emu 110: 364-377. https://doi.org/10.1071/MU10030

Wirdheim A (red). 2020. Sveriges fåglar 2020. BirdLife Sverige, Stockholm \& Svensk Fågeltaxering, Lunds universitet, Lund. 28 sid. Tillgänglig från https://www.fageltaxering.lu.se/sites/default/files/ files/Rapporter/sveriges_faglar_2020.pdf.

\section{English summary}

In birds, individuals with a mixed female and male plumage are reported regularly, including gynandromorphs and more gradually masculinized females with an intersex phenotype. The occurrence of intersex individuals is more easily detected in species with distinct plumage differences between males and females, such as in ducks, but should occur throughout the bird phylogeny. However, although detected regularly, only few reports of intersex individuals are described in the literature (but see Chiba \& Honma 2011).

In this short communication, we describe the plumage changes in a Mallard Anas platyrhynchos intersex individual, hatched 2014 or earlier, over six years (2015-2020), from a mixed female-male phenotype to a predominantly male phenotype. All observations were made by one of the authors (AX) in Umeå, Västerbotten, in northern Sweden, and photographs collected from encounters with the bird are presented in Figures 1-4. For links to all photographs, see Table 1.

Already at the first observation, the bird, although predominantly of female phenotype, had some typical male characteristics. Such traits included a whitish-grey tail with the two typical male central black curved rectrices, as well as a greenish tinge to parts of the head (Figure 1). After moult in summer 2015, the bird attained a more male phenotype plumage (Figure 2) and kept that plumage for the remainder of the ob- servation period (the bird is still present at the time of writing, January 2021). The plumage was never completely male; female-patterned body feathers remained, especially on the side of the body, until 2019, but in reduced frequency over time (Figures 2-4). Moreover, the cheeks and ear-coverts continued to display a variation in colour from white to green, and sometimes apparently with reduced feathering. Throughout the study period, the bill remained patterned as a female. The bird was never observed in eclipse plumage.

We do not know of the circumstances that lead to masculinization in this intersex individual, but generally such changes seem to occur if the hormone production of the ovary is severely impaired. This has been described in Northern Pintails Anas acuta in Japan, where intersex birds were found at a frequency of $0.01-0.18 \%$ in winter, and where dissection of a few intersex birds showed changes to the ovary (Chiba \& Honma 2011). The Northern Pintail study, and the Mallard presented here, indicate that change of sex plumage phenotype is most rapid in the beginning, and that later changes are slighter. We encourage all ornithologists to report intersex individuals and deposit their observations in one of the many online faunistic databases. In Table 2, a summary of other Mallards (and a Eurasian Teal Anas crecca) with plumage suggesting intersex, gathered from the Swedish Species Observation System (www.artportalen.se), is presented.

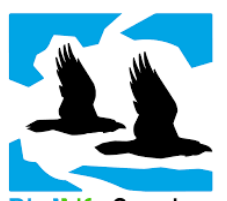

BirdLife Sverige

Ornis Svecica (ISSN 2003-2633) is an open access, peer-reviewed scientific journal published in English and Swedish by BirdLife Sweden. It covers all aspects of ornithology, and welcomes contributions from scientists as well as non-professional ornithologists. Accepted articles are published at no charge to the authors. Read papers or make a submission at os.birdlife.se.

Ornis Svecica (ISSN 2003-2633) är en fritt tillgänglig granskad vetenskaplig tidskrift som ges ut på svenska och engelska av BirdLife Sverige. Den täcker ornitologins alla områden och välkomnar bidrag från såväl forskare som icke-professionella ornitologer. Accepterade uppsatser publiceras utan kostnad för författarna. Läs uppsatser eller skicka in ditt bidrag på os.birdlife.se. 